\title{
A Systematic Review of Pediatric Telediabetes Service Models
}

\author{
Keshia R. De Guzman, BPharm, Centaine L. Snoswell, PhD, MPH, BPharm, ${ }^{1,2}$ \\ Monica L. Taylor, MPH, ${ }^{1,2}$ Buddhika Senanayake, MSC, MBBS, ${ }^{1,2}$ \\ Helen M. Haydon, PhD, Hons, BPsy, ${ }^{1,2}$ Jennifer A. Batch, FRACP, MBBS, \\ Anthony C. Smith, PhD, MEd, BN, RN, ${ }^{1,2}$ and Liam J. Caffery, PhD, Info Tech ${ }^{1,2}$
}

\begin{abstract}
Telediabetes may improve patient access to clinicians who specialize in the management of pediatric diabetes. Due to the diversity of telehealth modes, many different service models for pediatric telediabetes have been developed. This review describes pediatric telediabetes service models identified in the literature, investigates the reported changes in HbA1c of these interventions, and describes enablers and barriers to implementing a telediabetes service. Evaluation of current literature may inform the development and sustainability of telehealth services for pediatric diabetes management. Twenty-nine studies met inclusion criteria and were reviewed. This review has demonstrated that pediatric telediabetes can be delivered by remote monitoring and real-time videoconference modes. Overall, pediatric telediabetes increased interactions between patients and clinicians, improved access to specialized care, and facilitated increased diabetes monitoring. In some contexts, telediabetes also improved short-term glycemic control. Key enablers reported for telediabetes services were integration with existing workflows, dedicated staff, clinician and patient training, appropriate data security, technology with good usability, and the availability of technical support. Barriers included increases in patient responsibilities and clinician workload, and technical issues with equipment and software.
\end{abstract}

Keywords: Telemedicine, Telehealth, Telediabetes, Pediatric diabetes, Endocrinology.

\section{Introduction}

D IABETES CAN BE difficult to manage in the general population, and even more so in pediatric patients. ${ }^{1-5} \mathrm{Pe}-$ diatric patients undergo rapid changes in physical, cognitive, and social development. ${ }^{6}$ These changes increase the complexity of diabetes management. In addition, complicated family structures and family breakdown can lead to inconsistent management. ${ }^{7,8}$ Pediatric patients may have difficulty adhering to treatment plans that require frequent changes as the child grows and matures. ${ }^{3,9,10}$ The need for regular monitoring of blood glucose levels (BGLs) and complex insulin regimens (multiple daily injections and insulin pump therapy) may also be a challenge in this patient group. $5,11,12$
Children and adolescents often require support from parents, carers, or teachers, before they develop autonomy, and transition to diabetes self-management. ${ }^{13,14}$

Access to specialized services, regular monitoring, and individualized treatment are needed to achieve optimal diabetic control. ${ }^{11,15}$ There is an increasing need for diabetes services to support and treat pediatric patients to achieve glycemic targets and reduce long-term diabetic complications. ${ }^{10,15-17}$ However, there are limited numbers of clinicians who specialize in pediatric diabetes management, which may make it difficult for patients to receive care. ${ }^{15}$ Telehealth may be used to address some of the challenges associated with pediatric diabetes management. Telehealth has been shown to improve access to health care, facilitate

\footnotetext{
${ }^{1}$ Centre for Online Health, The University of Queensland, Brisbane, Australia.

${ }^{2}$ Centre for Health Services Research, The University of Queensland, Brisbane, Australia.

${ }^{3}$ Department of Endocrinology and Diabetes, Queensland Children's Hospital, Brisbane, Australia.

${ }^{4}$ Faculty of Medicine, The University of Queensland, Brisbane, Australia.
} 
clinician and patient communication, and improve diabetes monitoring and management. 5,18,19 Telehealth also encourages a multidisciplinary approach to care as patients can receive services from a variety of health professionals. 5,20

Real-time videoconference, store-and-forward (e.g., secure e-mail), and remote monitoring are common modes of telehealth. Remote monitoring (also commonly known as inhome monitoring and telemonitoring) involves the transmission of the patient's biometric data (e.g., BGLs), which is reviewed by a clinician who provides feedback or initiates an intervention to optimize BGLs. Due to the choice of different telehealth modes, a variety of service models for pediatric telediabetes have been developed.

No previous reviews have collated and synthesized available literature describing service models for pediatric telediabetes. Evaluation of this research may inform the development and sustainability of telehealth applications for pediatric diabetes management. Therefore, the aim of this study was to identify and describe pediatric telediabetes service models, investigate reported changes in HbAlc of these interventions, and identify enablers and barriers to service implementation.

\section{Materials and Methods}

This review was registered in the International Prospective Register of Systematic Reviews (PROSPERO: CRD42019141239). ${ }^{21}$

\section{Article identification}

Peer-reviewed articles published in English between January 2000 and June 2019 were identified using Boolean searches of the PubMed, EMBASE, and CINAHL databases. The search strategy included a combination of $\mathrm{MeSH}$ or Emtree terms and keywords related to pediatric telediabetes. The full strategy is available in the published PROSPERO protocol. $^{21}$

\section{Inclusion criteria}

Original research articles and study protocols were included if they described the service model for a pediatric telediabetes intervention. Therefore, this review involved a broad range of study types (such as experimental and observational). Articles were included if they described a telediabetes intervention for pediatric patients (or their carers) with diabetes (type 1 or type 2 ). Articles related to gestational diabetes or diabetic retinopathy were excluded due to their limited applicability to a pediatric population.

Pediatric patients were defined as subjects younger than 18 years when they commenced the intervention. Telediabetes services were defined as digitally enabled clinical services that incorporated interaction between the patient or carer and a clinician. For this reason, articles describing educational interventions or self-monitoring only were excluded. For the purpose of this review, telephone-only services, static websites, or stand-alone text messaging services were not considered telediabetes interventions. All other types of telediabetes technologies (e.g., real-time videoconference, remote monitoring, mHealth, and interactive web-based platforms) were included.

\section{Article selection}

Titles and abstracts found using the search strategy were screened independently by two authors (B.S. and M.L.T.). The full texts for these studies were obtained and independently assessed by two members of the research team (K.R.D.G. and M.L.T.) to further assess eligibility. Any study article where eligibility could not be agreed upon by (K.R.D.G. and M.L.T.) was assessed by a third and fourth reviewer (B.S. and C.L.S.).

\section{Data extraction and reporting}

Data were extracted from the full text articles. The following fields were included in the data extraction: country, study type, participant type, service model description (including the mode and type of technology), HbA1c results, and key findings. Each study type was also assigned a level of evidence, according to the National Health and Medical Research Council (NHMRC) hierarchy of evidence. ${ }^{22}$ Where required, authors were contacted to find information regarding the service model that was not described in the article. Synthesis of findings was narrative in keeping with the study aim of describing different service models. Findings were reported according to the Preferred Reporting Items for Systematic Reviews and Meta-Analyses (PRISMA) guidelines. $^{23}$

\section{Quality assessment}

The Standards for Quality Improvement Reporting Excellence (SQUIRE) 2.0 checklist was used to assess the reporting quality of each article. ${ }^{24}$ Each article was assigned a score out of 18 points. Higher scores indicated a higher reporting quality. SQUIRE content items included analysis of title, abstract, introduction, methods, results, discussion, conclusion, and other information. ${ }^{24}$ SQUIRE enabled a comparison of reporting quality across different study types identified in the included articles. Protocol articles were assigned a total possible score out of nine, as not all items were relevant to them.

\section{Results}

\section{Study selection}

In total, the literature search resulted in the selection of 29 studies that met the inclusion criteria. ${ }^{1-4,7,9,11,12,14-17,20,25-40}$ The search and screening process is shown in the PRISMA flow diagram (Supplementary Fig. S1).

\section{Study characteristics}

All studies described pediatric telediabetes service models (Table 1). Articles were published between 2000 and 2019. Studies were conducted in 13 different countries, with the majority of services from the United States $(n=15$, $52 \%)^{1,4,7,17,26-30,33-36,39,40}$ The most common study type was a randomized controlled trial (RCT) $(n=11$, $38 \%) .^{3,7,11,16,17,20,25,28,30,31,37}$ Most studies were conducted over 24 weeks $(n=9,31 \%),{ }^{1,4,15-17,20,26,27,31}$ with the minimum study length at 12 weeks, $3,12,26$ and the maximum study length at 240 weeks. ${ }^{37}$ There were two protocols included in this review. ${ }^{35,36}$ 


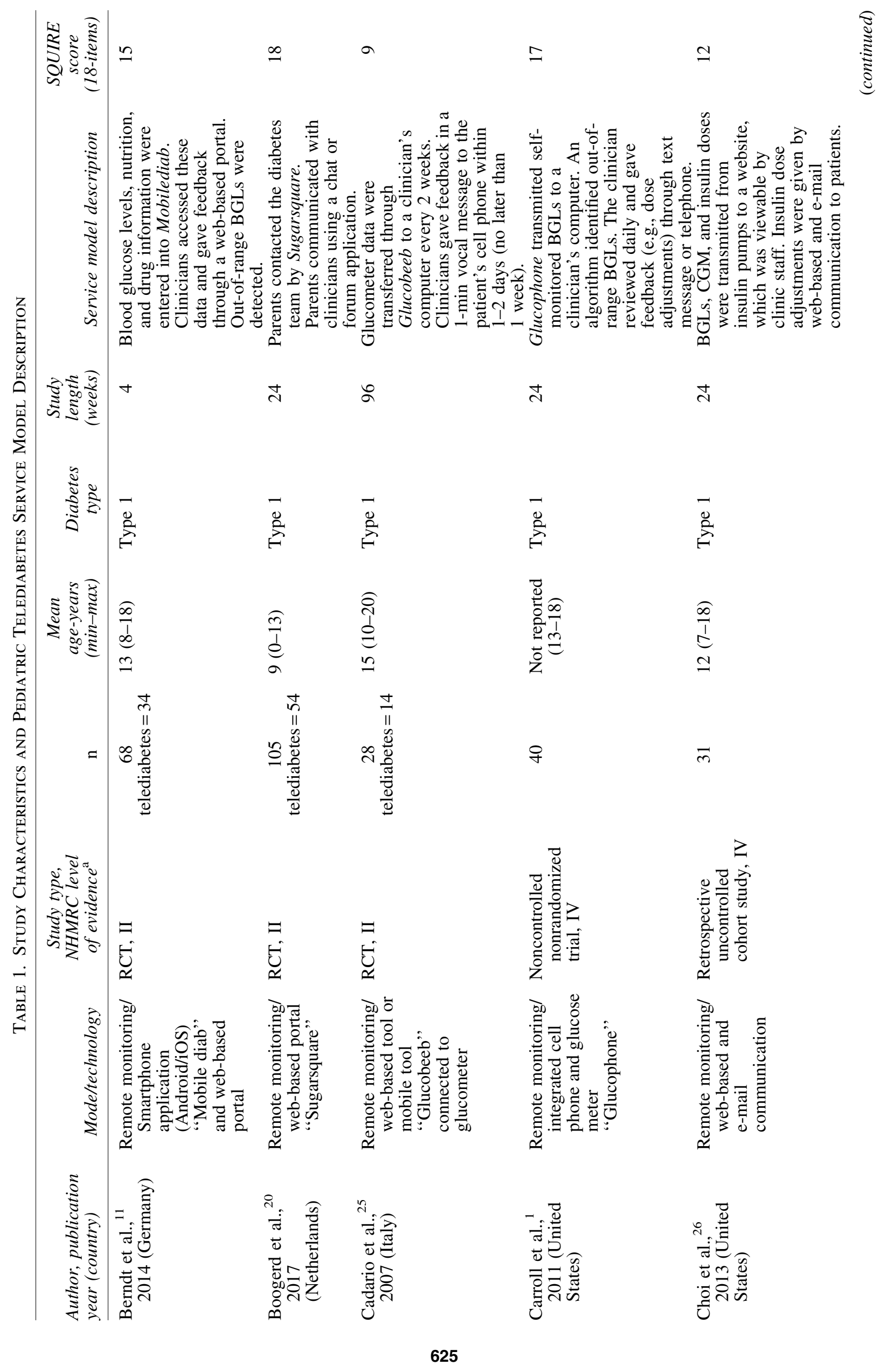




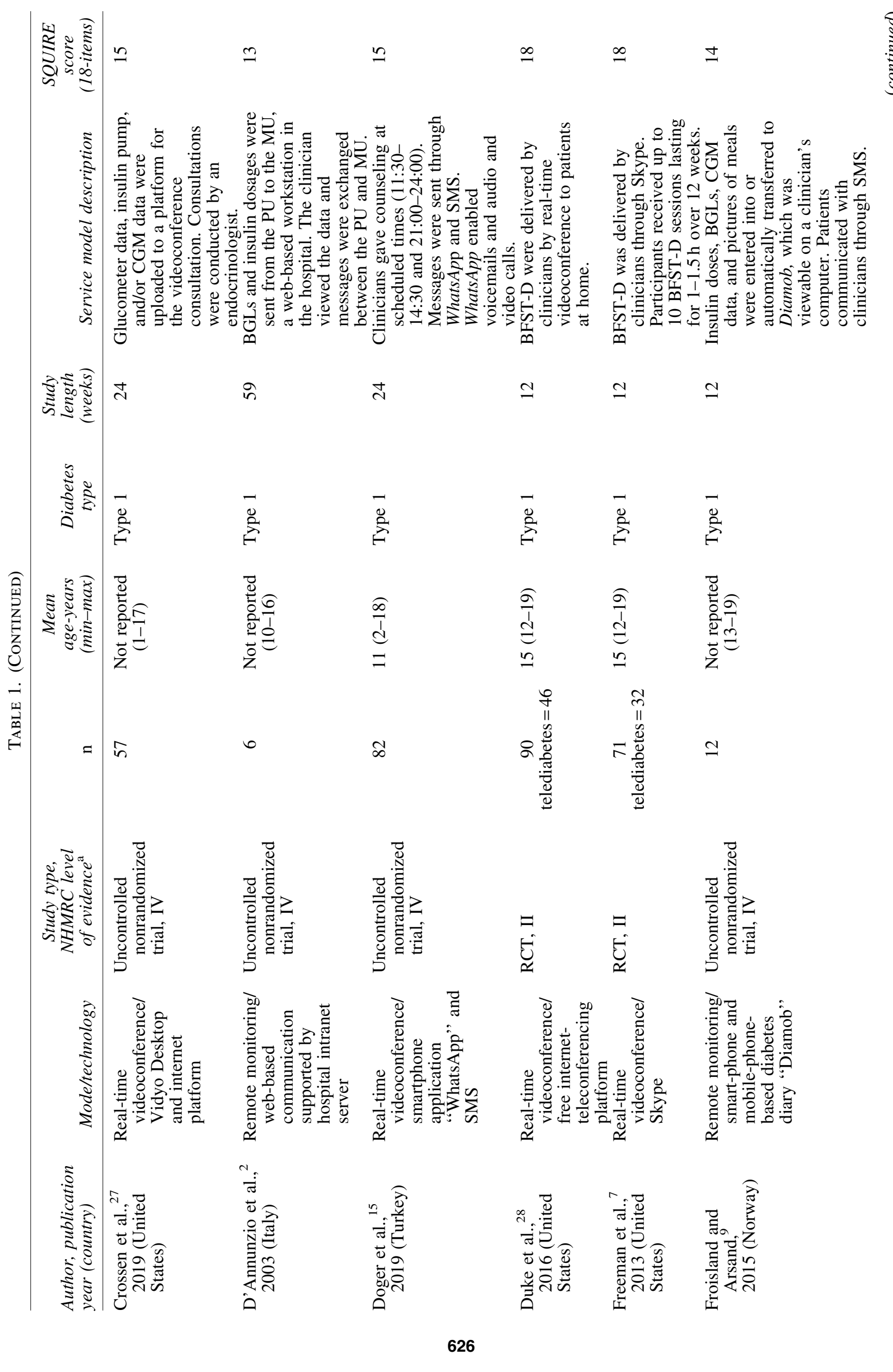




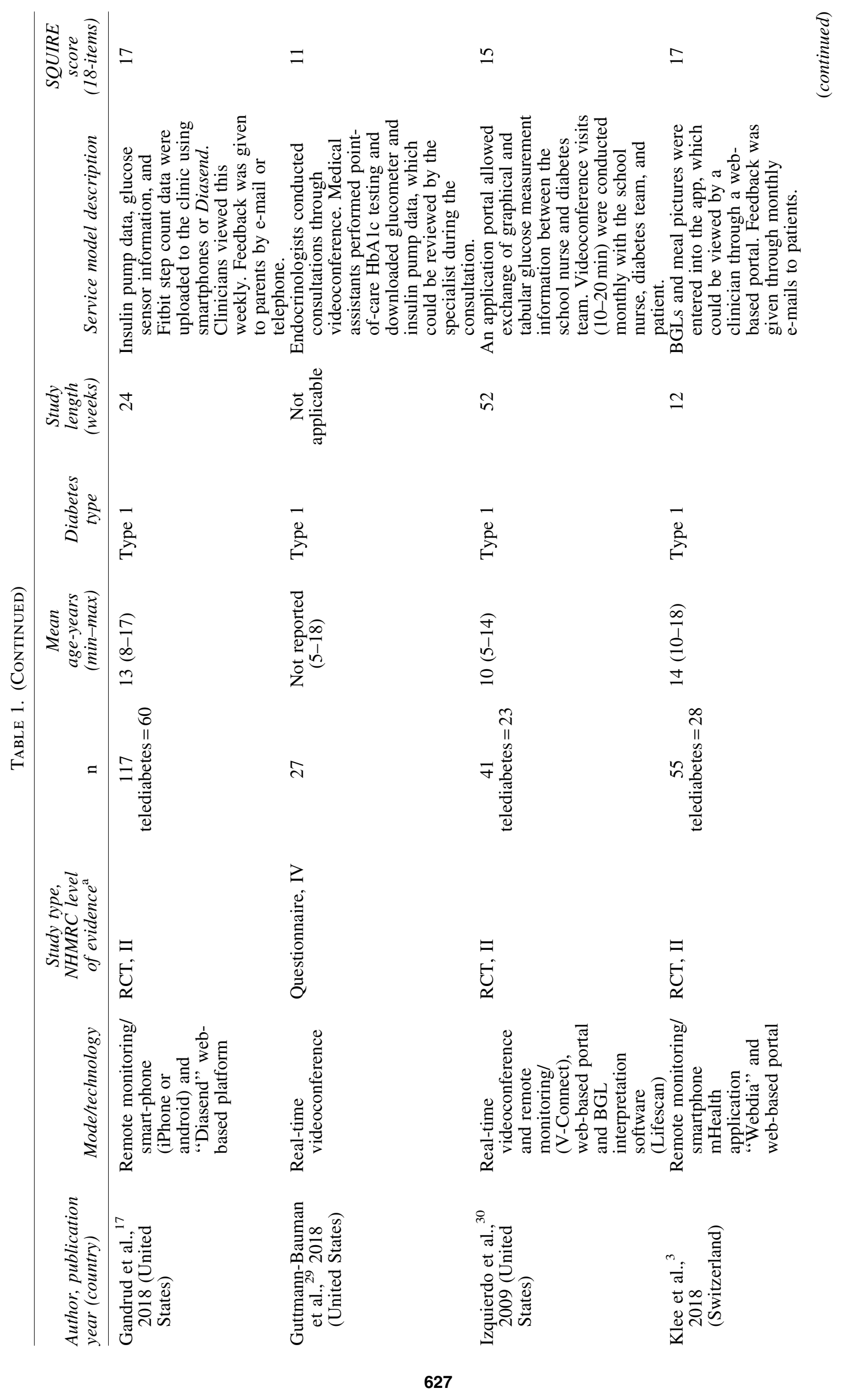




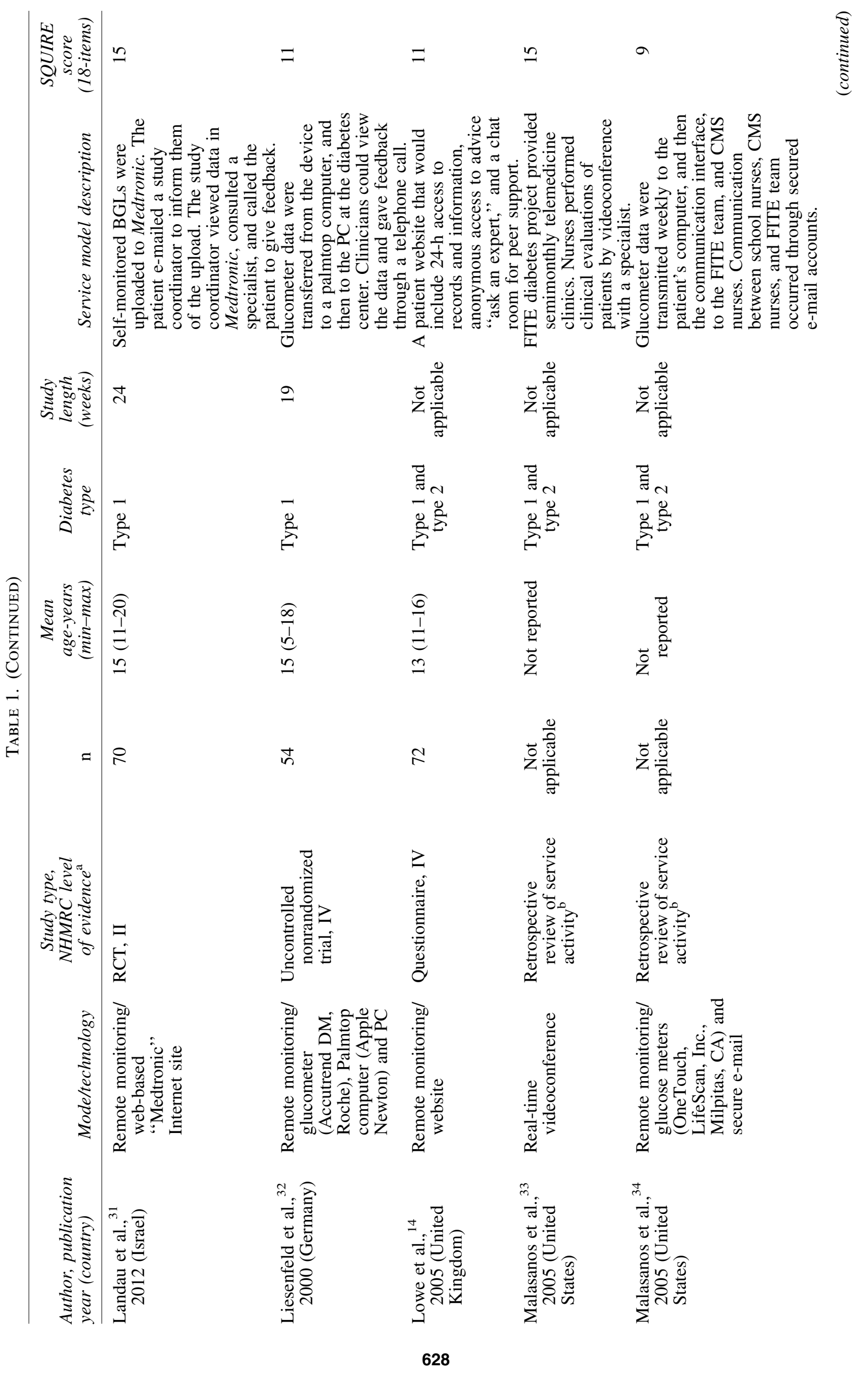




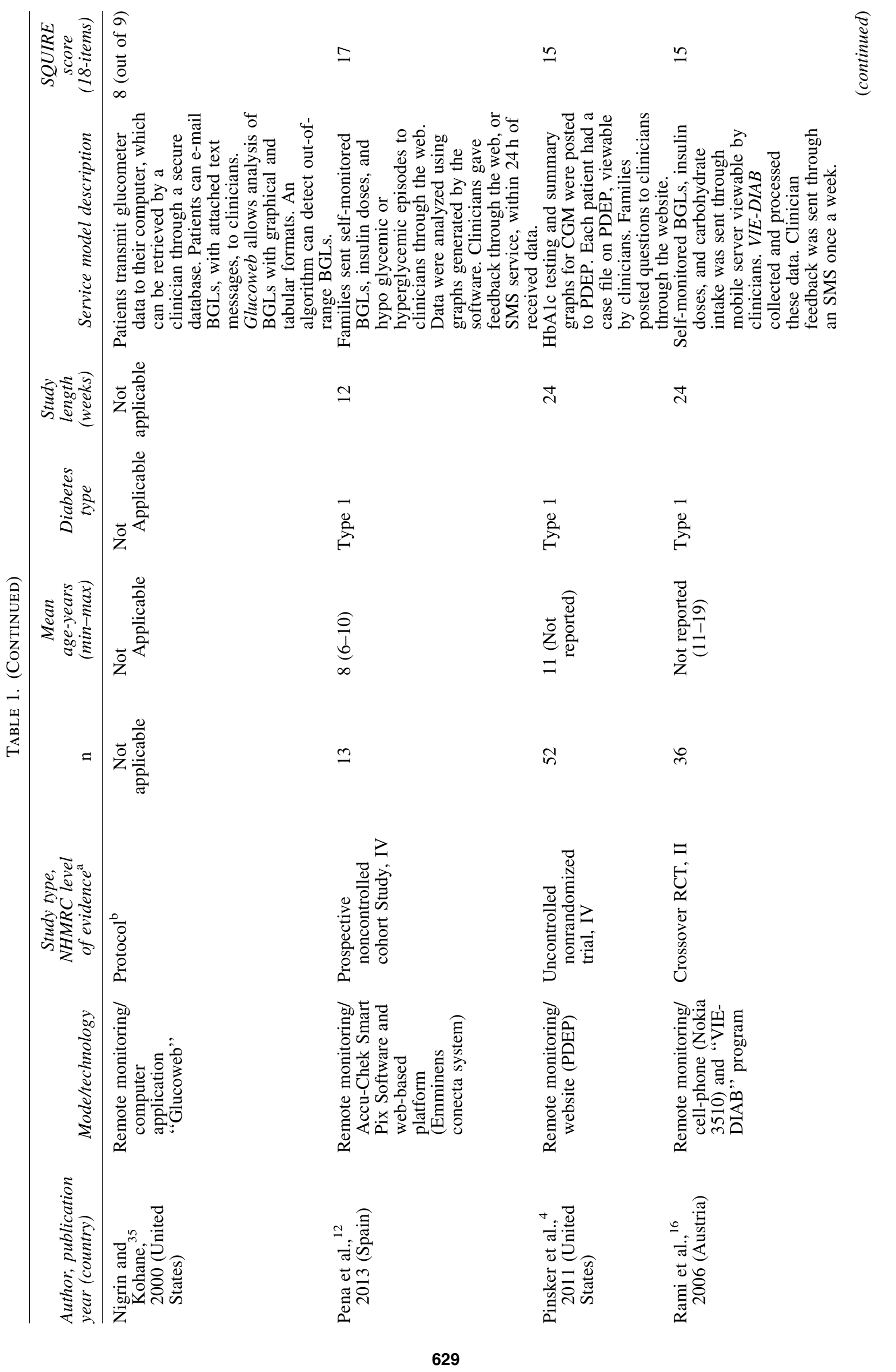




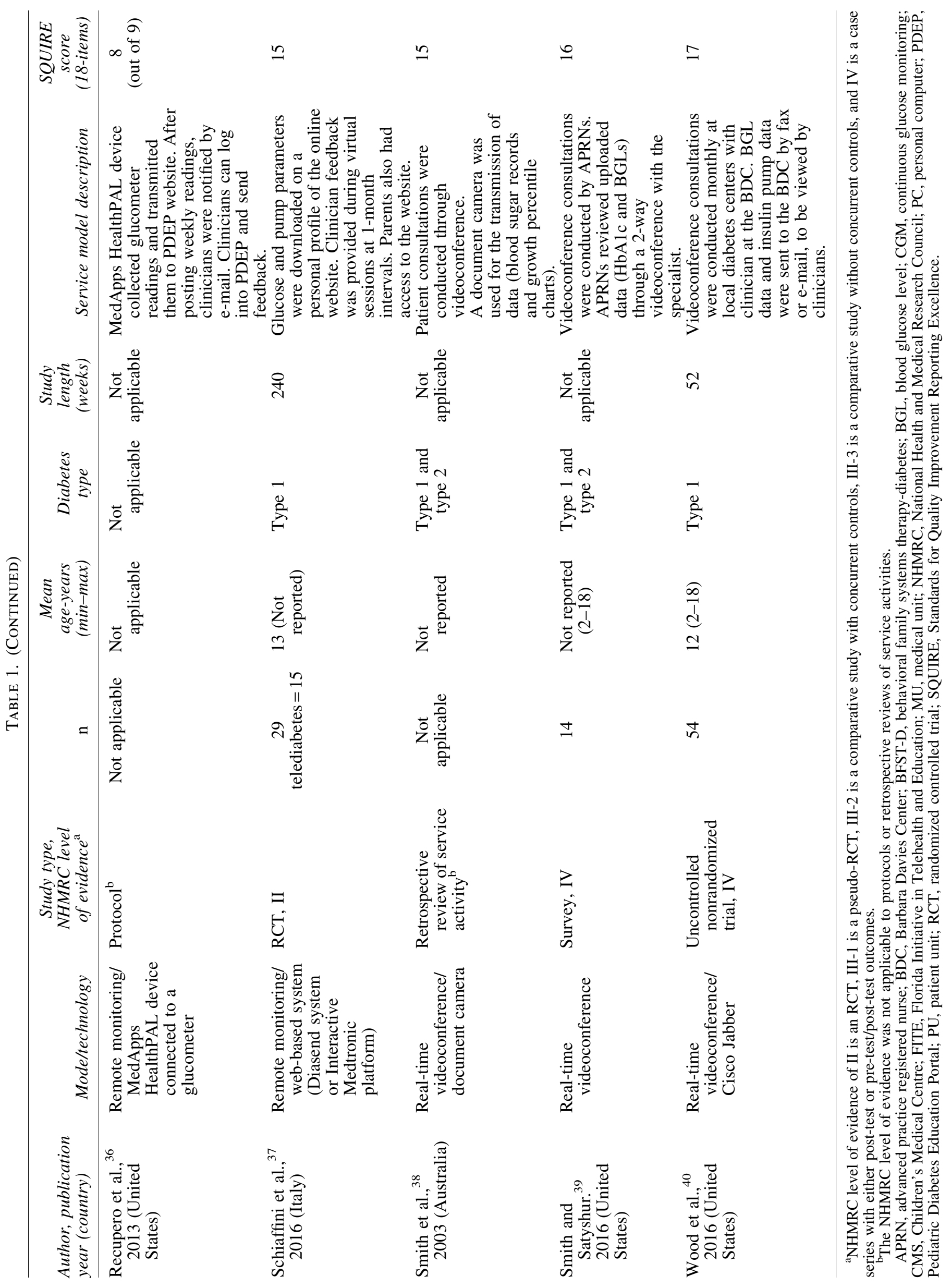




\section{Service models}

The majority of included studies used remote monitoring $(n=19,66 \%)$ as the main mode for delivering pediatric telediabetes services. ${ }^{1-4,9,11,12,14,16,17,20,25,26,31,32,34-37}$ Other modes included real-time videoconference $(n=9$, $31 \%)^{7,15,27-29,33,38-40}$ or a combination of remote monitoring and real-time videoconference modes $(n=1,3 \%){ }^{30}$ Storeand-forward was not used by any of the included studies. The service models utilized ancillary technologies, including mobile applications and web-based platforms. Some remote monitoring services had algorithms to alert out-of-range BGLs, which facilitated clinical review of the patient. ${ }^{1,11,35}$ Direct engagement between local clinicians and specialists during real-time videoconference sessions also led to training and educational opportunities. ${ }^{33,38,39}$

\section{Study reporting quality}

The median SQUIRE score for all included articles was 14, demonstrating an overall high quality of reporting. Three articles $(10 \%)$ were allocated a complete score of $18 .^{7,20,28}$ The main reasons for reductions in score were failure to adequately report certain items such as the rationale, reliability, and validity of measures $(n=14,48 \%)^{2,14-16,25-27,29,33-38}$; study limitations $(n=13,45 \%)^{2,4,9,11,14,25,32-38}$; and the context for the intervention $(n=12,41 \%) .^{2,4,12,16,17,25,27,31,32,35-37}$

\section{Clinical effectiveness}

Seventeen studies examined HbAlc (Table 2), and some of these studies also reported additional outcome measures. ${ }^{2-4,11,12,15-17,25-27,29-32,37,40}$ The remaining studies did not report $\mathrm{HbA} 1 \mathrm{c}$ and focused on satisfaction, feasibility, psychosocial effectiveness, usability, or a combination of these (Table 2). The results for change in HbA1c varied, making it difficult to determine the clinical effectiveness of pediatric telediabetes. Out of the 17 studies that examined HbA1c, 9 studies (53\%) were RCTs. ${ }^{3,4,11,16,17,25,30,31,37}$ The other eight studies $(47 \%)$ examined pediatric telediabetes

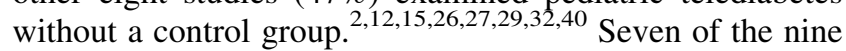
RCTs $(78 \%)$ reported improvements in HbA1c in the telediabetes group compared to the control group. $3,4,16,17,25,30,37$ The remaining two RCTs $(22 \%)$ reported no significant differences between groups. ${ }^{11,31}$

Out of the eight studies without a control group, five studies $(63 \%)^{12,15,26,27,32}$ reported HbA1c improvements and three studies $(38 \%)^{2,29,40}$ reported no significant change in $\mathrm{HbA1c}$. None of the included studies reported worsening of $\mathrm{HbA1c}$ as a result of the telediabetes intervention. D'Annunzio et al., ${ }^{2}$ Izquierdo et al., ${ }^{30}$ and Pena et al. ${ }^{12}$ did not report $\mathrm{HbA1c}$ values so the clinical effect of the telediabetes intervention could not be determined. The majority of studies measured HbAlc after participants had received the telediabetes intervention for 6 months. Although there were mixed $\mathrm{HbA} 1 \mathrm{c}$ results, pediatric telediabetes was found to be feasible, ${ }^{16,20,27,30}$ and most patients, families, and carers expressed satisfaction with these service models. $1,4,14,17,27,29,39,40$

\section{Enablers and barriers}

Enablers and barriers associated with the delivery of pediatric telediabetes services were identified across service models (Table 3 ). Enablers were patient and clinician training 7,12,25,29-31,33,34,37-39; secure technology and subsequent data confidentiality ${ }^{11,20,34}$; increased usability and convenience of the technology; and delegation of a team member to coordinate the telediabetes service. ${ }^{20}$ The most common barriers included technical difficulties, $1,2,4,15,30,40$ concern over data security, ${ }^{34}$ lack of reimbursement for telehealth activities, ${ }^{29,30,40}$ and the potential for increased patient responsibilities and clinician workload. ${ }^{1,2,16}$

\section{Discussion}

This review identified and described 29 pediatric telediabetes service models along with enablers and barriers to implementing these services. Remote monitoring (66\%) and real-time videoconference $(31 \%)$ were the most common mode of delivering pediatric telediabetes services. HbA1c was investigated by 17 of the included studies. Overall, the findings were mixed, with $78 \%$ of randomized controlled studies and $63 \%$ of noncontrolled studies reporting improved glycemic control for telediabetes. For the remaining studies, there was no significant change between telediabetes and standard care (in-person consultations). No study reported worsening of $\mathrm{HbAlc}$.

Differences in patient's age, sociodemographic characteristics, baseline HbA1c, diabetes type, stage of disease, insulin regimen, and BGL monitoring may explain variability in reported findings. For example, enrolment eligibility was different for each study with duration of diabetes required to be over 6 months for Landau et al. ${ }^{31}$; over 1 year for Rami et al. ${ }^{16}$ and Klee et al. ${ }^{3}$; and over 2 years for Cadario et al. ${ }^{25}$ Many of the included studies were examined with the short-term benefits, which raises the question as to whether the finding of $\mathrm{HbA} 1 \mathrm{c}$ improvement truly reflects long-term benefit.

In short-term follow-ups, patients may modify their behavior due to their awareness that they are being monitored. For instance, Gandrud et al. ${ }^{17}$ found HbA1c improvement was not sustained when measured 3 months postintervention, while Pena et al. ${ }^{12}$ reported worsening of glycemic control after cessation of the telediabetes intervention. Conversely, Schiaffini et al. ${ }^{37}$ followed patients for 5 years; however, they only included sensor-augmented pump-treated adolescents, and their results may not be applicable to patients using different insulin regimens (e.g., multiple daily injections) with self-monitored BGLs.

This review demonstrated that pediatric telediabetes increased interactions between patients and clinicians, resulting in improved access to specialized care and efficient diabetes monitoring. Increased clinician interaction and additional support for diabetes management are particularly important for pediatric patients. ${ }^{13,14}$ A multidisciplinary approach was encouraged by some services. ${ }^{20,30,34,39,40}$ This was a positive outcome because pediatric patients were able to access a variety of health professionals and clinical services. Other service models created a supportive network of care by involving diabetes teams, clinicians, school nurses, and caregivers. ${ }^{30,34}$

Successful service models were found to be integrated with existing health care systems. Boogerd et al. ${ }^{20}$ highlighted that a designated telehealth team member helped integrate their service into practice. Shared workload, established partnerships, and a common goal were identified as key enablers of 


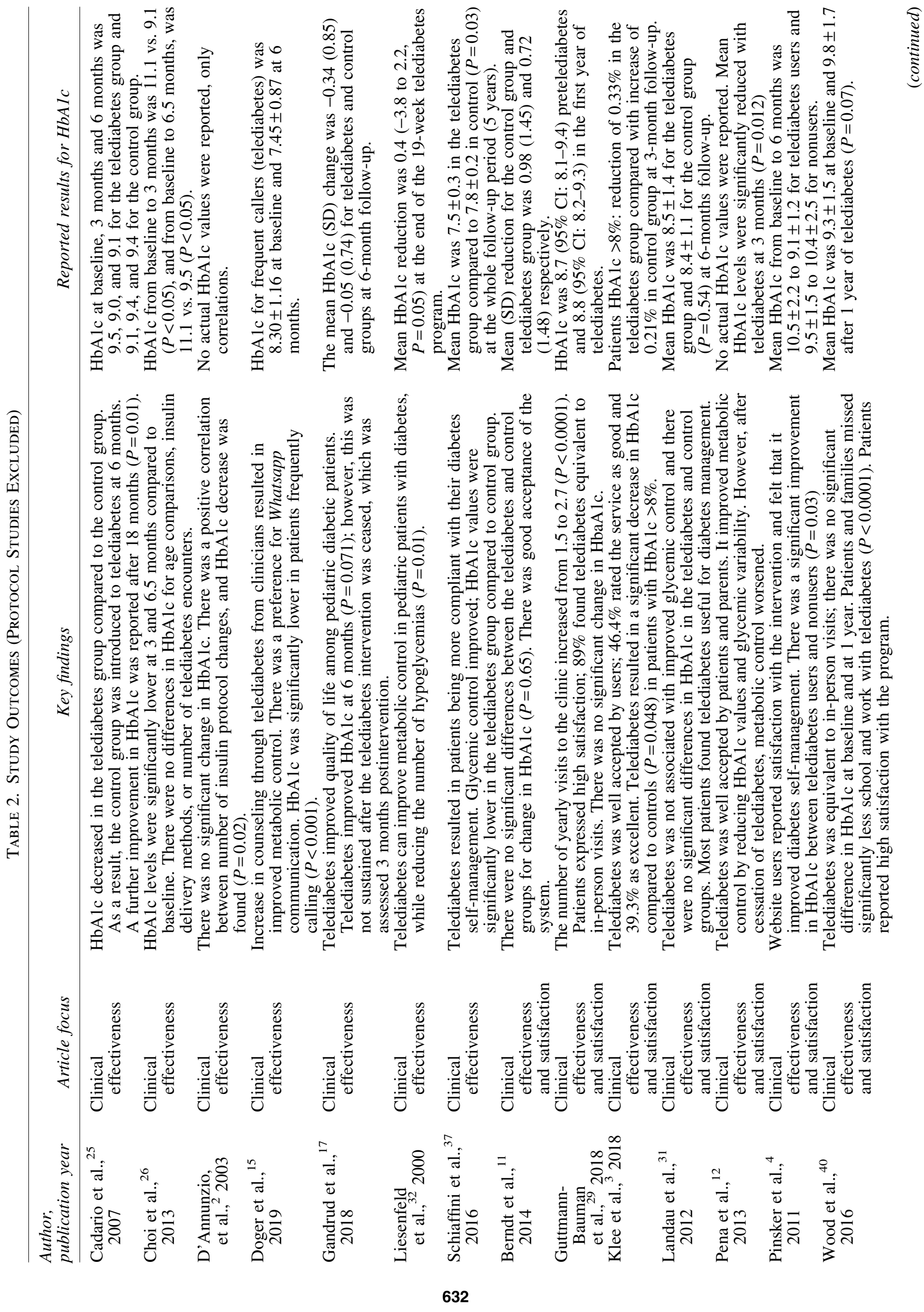




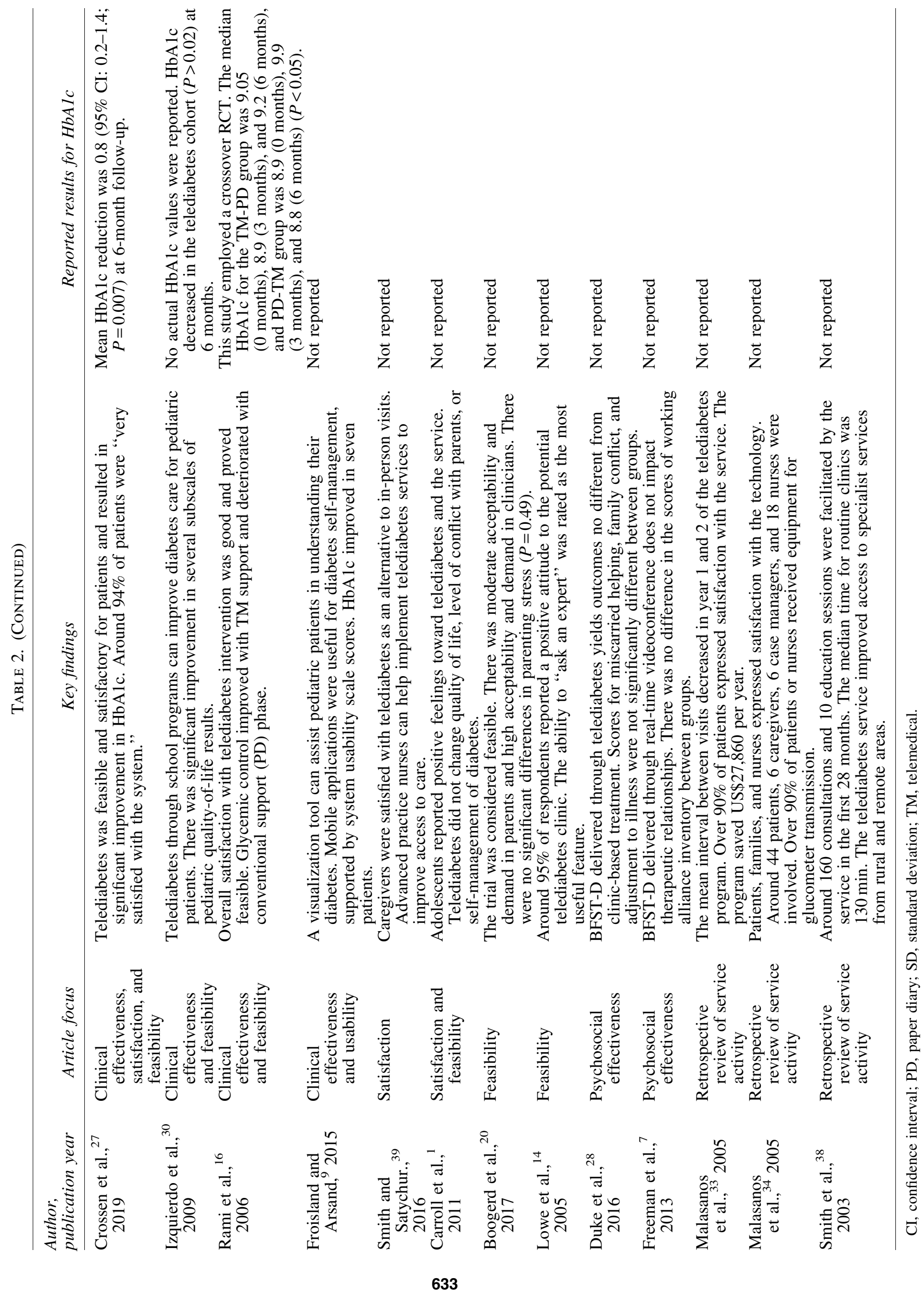




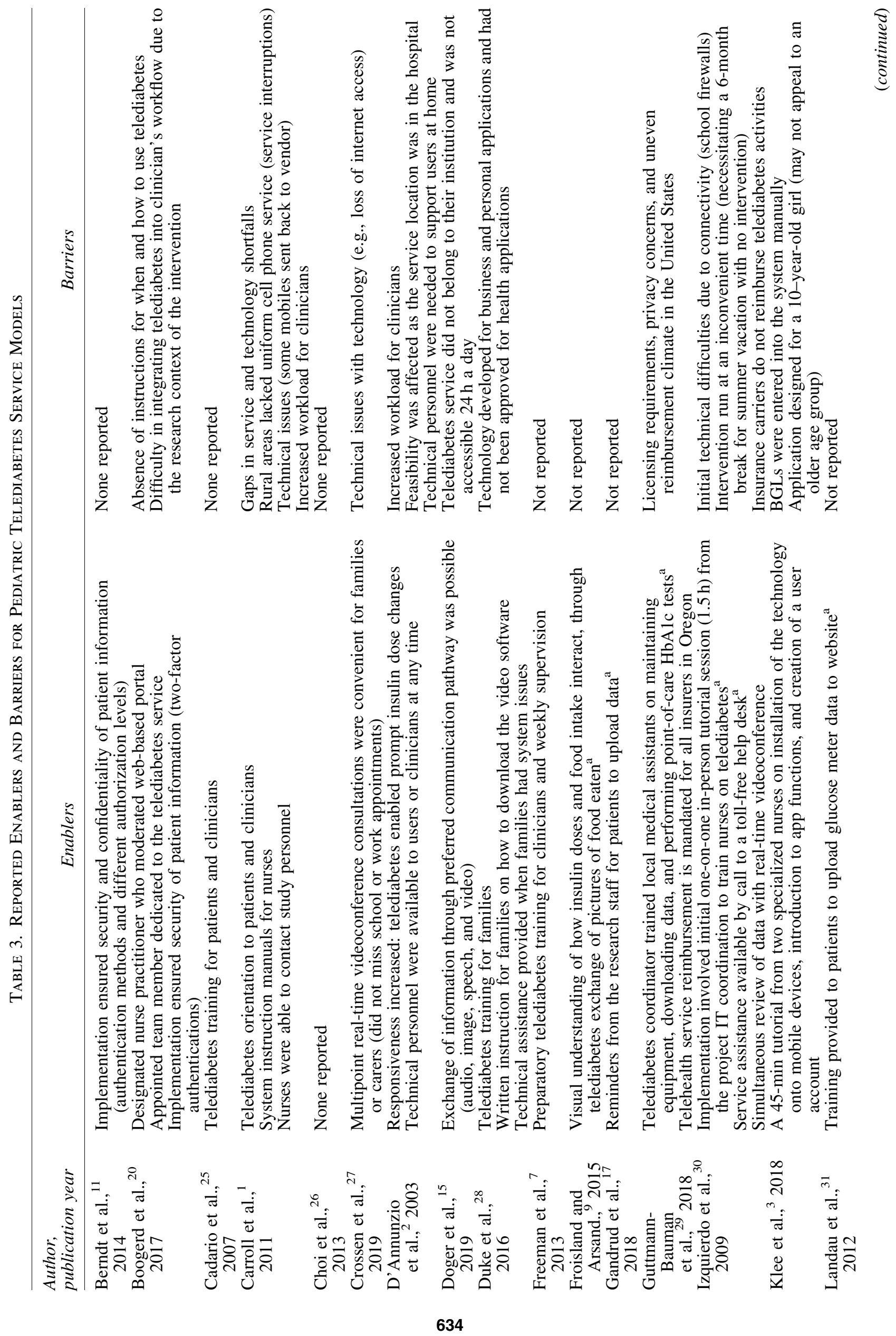




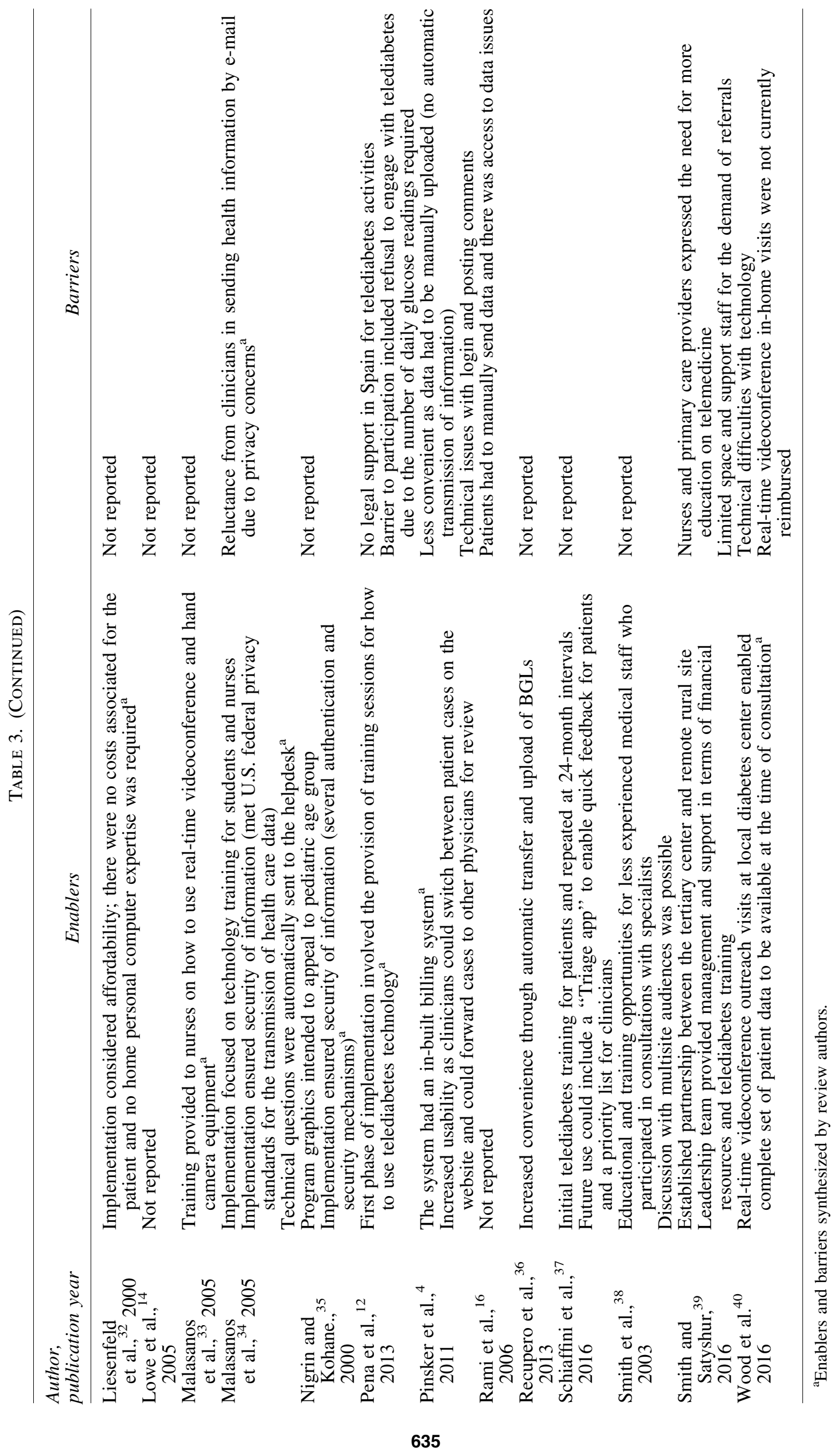


successful implementation of pediatric telediabetes. ${ }^{20,39}$ Service models need to be convenient and have good usability to reduce the burden on patients and clinicians. Recupero et al. ${ }^{36}$ improved the functionality of an intervention by enabling the automatic transfer of BGLs, rather than manual data entry. Telediabetes services, which included training for clinicians and patients, demonstrated the importance of ensuring that patients and clinicians understand the technology. In addition, having organizational support, technical support, and management assistance were considered essential for successful service implementation.

Telediabetes services also reported various challenges; technical issues negatively influenced patients' willingness to engage in pediatric telediabetes. ${ }^{1,27}$ Telediabetes services also impacted on clinicians' workflows and Carrol et al. ${ }^{1}$ reported that nurse practitioners were giving feedback to patients outside of usual clinical hours. Others reported that telediabetes services were offered in addition to standard care, resulting in increased clinician workload. ${ }^{2,20}$ This was often in the absence of recognition from health services of the work and time involved with telediabetes, as well as funding for additional staff or appropriate remuneration. ${ }^{29,30,40}$

There have been three other reviews of pediatric telediabetes. ${ }^{41-43}$ Our review was of broader scope as it included both type 1 and type 2 patients. The reviews by Guljas et al. ${ }^{41}$ and Shulman et al. ${ }^{43}$ were limited to type 1 patients. Furthermore, we included all modes of telediabetes, whereas Deacon and Edirippulige ${ }^{42}$ only evaluated mHealth interventions and Shulman et al. ${ }^{43}$ only examined remote monitoring.

We also identified key enablers and barriers to telediabetes service implementation, which was not discussed by Deacon and Edirippulige, ${ }^{42}$ Guljas et al., ${ }^{41}$ and Shulman et al. ${ }^{43}$ Guljas et al. ${ }^{41}$ concluded that telediabetes improved management of type 1 diabetes in pediatric patients. Shulman et al. ${ }^{43}$ conducted a meta-analysis of pediatric telediabetes on glycemic control, finding that although telediabetes did not significantly improve HbAlc, it did not worsen glycemic control. We found that change in HbAlc results varied across studies, comparable with the findings by Deacon and Edirippulige, ${ }^{42}$ who reported mixed results for clinical effectiveness.

\section{Implications for practice}

It is important to understand how various pediatric telediabetes service models can best be integrated into routine clinical practice. The enablers and barriers identified in this review outlined logistical factors associated with the delivery of the service and the importance of ensuring that telediabetes services complemented conventional services. Remote monitoring was feasible, and the most common mode used, achieving improved diabetes monitoring. Realtime videoconference modes improved a patient's access to care, particularly given the limited number of health professionals who specialize in pediatric diabetes management. It has been demonstrated that pediatric telediabetes does not compromise clinical care as telediabetes did not worsen glycemic control.

\section{Limitations}

Many of the included studies had a short-term study period. The finding of HbA1c improvement requires long-term follow-up to assess the sustainability of results. Some of the included studies also had small sample sizes, reducing the power of their results. Given that the articles were published between 2000 and 2019, the change in technology overtime needs to be acknowledged. We can draw no conclusion as to whether pediatric telediabetes is sustainable as there are no long-term studies of any service. Publication bias is also recognized as studies may be more likely to report positive outcomes favoring telehealth.

\section{Conclusion}

This review has demonstrated that pediatric telediabetes can be delivered by remote monitoring and real-time videoconference modes. The use of pediatric telediabetes increased interactions between patients and clinicians, improving access to specialized care and diabetes monitoring. Most patients, families, and caregivers expressed satisfaction with telediabetes services. In some situations, telediabetes improved glycemic control in the short term. Further research is still required to determine the long-term clinical benefit of telediabetes. Additional investigation into the costeffectiveness of telediabetes will also have to be considered for future research.

Integration of telediabetes services with existing clinical workflows, dedicated staff, clinician and patient training, appropriate data security, technology with good usability, and the availability of technical support, would enable the positive outcomes of telediabetes services. Increased patient responsibilities and clinician workload, and technical issues are likely to act as barriers to telediabetes services.

\section{Author Disclosure Statement}

No competing financial interests exist.

\section{Funding Information}

No funding was received for this article.

\section{Supplementary Material}

Supplementary Figure S1

\section{References}

1. Carroll AE, DiMeglio LA, Stein S, et al.: Using a cell phone-based glucose monitoring system for adolescent diabetes management. Diabetes Educ 2011;37:59-66.

2. D'Annunzio G, Bellazzi R, Larizza C, et al.: Telemedicine in the management of young patients with type 1 diabetes mellitus: a follow-up study. Acta Biomed 2003;74(Suppl. 1): 49-55.

3. Klee P, Bussien C, Castellsague M, et al.: An intervention by a patient-designed do-it-yourself mobile device app reduces HbA1c in children and adolescents with type 1 diabetes: a randomized double-crossover study. Diabetes Technol Ther 2018;20:797-805.

4. Pinsker JE, Nguyen C, Young S, et al.: A pilot project for improving paediatric diabetes outcomes using a website: the Pediatric Diabetes Education Portal. J Telemed Telecare 2011;17:226-230.

5. Raymond JK: Models of care for adolescents and young adults with type 1 diabetes in transition: shared medical appointments and telemedicine. Pediatr Ann 2017;46: e193-e197. 
6. Halvorson M, Yasuda P, Carpenter S, et al.: Unique challenges for pediatric patients with diabetes. Diabetes Spectr 2005;18:167-173.

7. Freeman KA, Duke DC, Harris MA: Behavioral health care for adolescents with poorly controlled diabetes via Skype: does working alliance remain intact? J Diabetes Sci Technol 2013;7:727-735.

8. Leonard BJ, Garwick A, Adwan JZ: Adolescents' perceptions of parental roles and involvement in diabetes management. J Pediatr Nurs 2005;20:405-414.

9. Froisland DH, Arsand E: Integrating visual dietary documentation in mobile-phone-based self-management application for adolescents with type 1 diabetes. J Diabetes Sci Technol 2015;9:541-548.

10. Gay CL, Chapuis F, Bendelac N, et al.: Reinforced followup for children and adolescents with type 1 diabetes and inadequate glycaemic control: a randomized controlled trial intervention via the local pharmacist and telecare. Diabetes Metab 2006;32:159-165.

11. Berndt RD, Takenga $C$, Preik $P$, et al.: Impact of information technology on the therapy of type-1 diabetes: a case study of children and adolescents in Germany. J Pers Med 2014;4:200-217.

12. Pena NV, Torres M, Cardona JA, et al.: Impact of telemedicine assessment on glycemic variability in children with type 1 diabetes mellitus. J Diabetes Sci Technol 2013; 15:136-142.

13. Curtis J, Hagerty D: Managing diabetes in childhood and adolescence. Can Fam Phys 2002;48:499-509.

14. Lowe P, Hearnshaw H, Griffiths F: Attitudes of young people with diabetes to an Internet-based virtual clinic. J Telemed Telecare 2005;11(Suppl. 1):59-60.

15. Doger E, Bozbulut R, Soysal Acar AS, et al.: Effect of telehealth system on glycemic control in children and adolescents with type 1 diabetes. J Clin Res Pediatr Endocrinol 2019;11:70-75.

16. Rami B, Popow C, Horn W, et al.: Telemedical support to improve glycemic control in adolescents with type 1 diabetes mellitus. Eur J Pediatr 2006;165:701-705.

17. Gandrud L, Altan A, Buzinec P, et al.: Intensive remote monitoring versus conventional care in type 1 diabetes: a randomized controlled trial. Pediatr Diabetes 2018;19: 1086-1093.

18. Dougherty JP, Lipman TH, Hyams S, et al.: Telemedicine for adolescents with type 1 diabetes. West J Nurs Res 2014; 36:1199-1221.

19. Smith AC, Scuffham P, Wootton R: The costs and potential savings of a novel telepaediatric service in Queensland. BMC Health Serv Res 2007;7:35.

20. Boogerd E, Maas-Van Schaaijk NM, Sas TC, et al.: Sugarsquare, a web-based patient portal for parents of a child with type 1 diabetes: multicenter randomized controlled feasibility trial. J med Internet Res 2017;19: e287.

21. Snoswell CL, Smith AC, Caffery LJ, et al.: Models of care in paediatric telediabetes. PROPSERO 2019 CRD42019141239. 2019. https://www.crd.york.ac.uk/ prospero/display_record.php?ID=CRD42019141239 (accessed November 1, 2019).

22. Shekelle PG, Maglione MA, Luoto J, et al.: Global health evidence evaluation framework [Internet]. Tablet B.9, NHMRC evidence hierarch: designations of 'levels of evidence' according to the type of research question (in- cluding explanatory notes). https://www.ncbi.nlm.nih.gov/ books/NBK121300/table/appb.t21/ (accessed December 4, 2019).

23. Moher D, Liberati A, Tetzlaff J, et al.: Preferred reporting items for systematic reviews and meta-analyses: the PRISMA statement. Ann Intern Med 2009;51:264-269.

24. Ogrinc G, Davies L, Goodman D, et al:: SQUIRE 2.0 (Standards for QUality Improvement Reporting Excellence): revised publication guidelines from a detailed consensus process. BMJ Qual Saf 2016;25:986-992.

25. Cadario F, Binotti M, Brustia M, et al.: Telecare for teenagers with type 1 diabetes: a trial. Minerva Pediatr 2007;59: 299-305.

26. Choi YS, Berry-Caban C, Nance J: Telemedicine in paediatric patients with poorly controlled type 1 diabetes. J Telemed Telecare 2013;19:219-221.

27. Crossen S, Glaser N, Sauers-Ford H, et al.: Home-based video visits for pediatric patients with poorly controlled type 1 diabetes. J Telemed Telecare 2019 [Epub ahead of print]; DOI: 10.1177/1357633X19828173.

28. Duke DC, Wagner DV, Ulrich J, et al.: Videoconferencing for teens with diabetes: family matters. J Diabetes Sci technol 2016;10:816-823.

29. Guttmann-Bauman I, Kono J, Lin AL, et al.: Use of telehealth videoconferencing in pediatric type 1 diabetes in Oregon. Telemed J E Health 2018;24:86-88.

30. Izquierdo R, Morin PC, Bratt K, et al.: School-centered telemedicine for children with type 1 diabetes mellitus. J Pediatr 2009;155:374-379.

31. Landau Z, Mazor-Aronovitch K, Boaz M, et al.: The effectiveness of Internet-based blood glucose monitoring system on improving diabetes control in adolescents with type 1 diabetes. Pediatr Diabetes 2012;13:203-207.

32. Liesenfeld B, Renner R, Neese M, et al.: Telemedical care reduces hypoglycemias and improves glycemic control in children and adolescents with type 1 diabetes. Diabetes Technol Ther 2000;2:561-567.

33. Malasanos TH, Burlingame JB, Youngblade L, et al.: Improved access to subspecialist diabetes care by telemedicine: cost savings and care measures in the first two years of the FITE diabetes project. J Telemed Telecare 2005;11(Suppl. 1):74-76.

34. Malasanos TH, Patel BD, Klein J, et al.: School nurse, family and provider connectivity in the FITE diabetes project. J Telemed Telecare 2005;11(Suppl. 1): 76-78.

35. Nigrin DJ, Kohane IS: Glucoweb: a case study of secure, remote biomonitoring and communication. Proc AMIA Symp 2000:610-614.

36. Recupero A, Mahnke B, Pinsker JE: Emerging technology in diabetes care: the real-time diabetes monitoring system. Mil Med 2013;178:218-221.

37. Schiaffini R, Tagliente I, Carducci C, et al.: Impact of longterm use of eHealth systems in adolescents with type 1 diabetes treated with sensor-augmented pump therapy. J Telemed Telecare 2016;22:277-281.

38. Smith AC, Batch J, Lang E, et al.: The use of online health techniques to assist with the delivery of specialist paediatric diabetes services in Queensland. J Telemed Telecare 2003; 9(Suppl. 2):S54-S57.

39. Smith NM, Satyshur RD: Pediatric diabetes telemedicine program improves access to care for rural families: role of APRNs. Pediatr Nurs 2016;42:294-299. 
40. Wood CL, Clements SA, McFann K, et al.: Use of telemedicine to improve adherence to American Diabetes Association standards in pediatric type 1 diabetes. Diabetes Technol Ther 2016;18:7-14.

41. Guljas R, Ahmed A, Chang K, et al.: Impact of telemedicine in managing type 1 diabetes among school-age children and adolescents: an integrative review. J Pediatr Nurs 2014;29:198-204.

42. Deacon AJ, Edirippulige S: Using mobile technology to motivate adolescents with type 1 diabetes mellitus: a systematic review of recent literature. J Telemed Telecare 2015;21:431-438.

43. Shulman RM, O'Gorman CS, Palmert MR: The impact of telemedicine interventions involving routine transmission of blood glucose data with clinician feedback on metabolic control in youth with type 1 diabetes: a systematic review and meta-analysis. Int J Pediatr Endocrinol 2010;2010: 536957.

Address correspondence to: Anthony C. Smith, PhD, MEd, BN, RN Centre for Online Health The University of Queensland Ground Floor Building 33 Princess Alexandra Hospital, Woolloongabba Brisbane 4102 Queensland Australia

E-mail: asmith@uq.edu.au 